\title{
A Fast Finite-Difference Time Domain Simulation Method for the Source-Stirring Reverberation Chamber
}

\author{
Wenxing Li, ${ }^{1}$ Chongyi Yue, ${ }^{1}$ and Atef Elsherbeni ${ }^{2}$ \\ ${ }^{1}$ Department of Information and Communication Engineering, Harbin Engineering University, Harbin, Heilongjiang 150001, China \\ ${ }^{2}$ College of Engineering and Computational Sciences, Colorado School of Mines, Golden, CO 80401, USA \\ Correspondence should be addressed to Chongyi Yue; yuechongyi@hrbeu.edu.cn
}

Received 19 December 2016; Accepted 13 February 2017; Published 8 March 2017

Academic Editor: Safieddin Safavi-Naeini

Copyright (c) 2017 Wenxing Li et al. This is an open access article distributed under the Creative Commons Attribution License, which permits unrestricted use, distribution, and reproduction in any medium, provided the original work is properly cited.

\begin{abstract}
Numerical analysis methods are often employed to improve the efficiency of the design and application of the source-stirring reverberation chamber. However, the state of equilibrium of the field inside the chamber is hard to reach. In this paper, we present a fast simulation method, which is able to significantly decrease the simulation time of the source-stirring reverberation chamber. The mathematical model of this method is given in detail and home-made FDTD code is employed to conduct the simulations and optimizations as well. The results show that the implementation of the method can give us the accurate frequency response of the source-stirring chamber and make the simulation of source-stirring chamber more efficient.
\end{abstract}

\section{Introduction}

Reverberation chamber (RC) is a kind of test facility for EMC, EMI, and antenna measurements [1-4]. As a new type of RC, the source-stirring reverberation chamber (SSRC) was proposed recently and attracted much notice [5-8]. The FDTD method is a suitable simulation tool of RCs [9-11], especially for SSRCs since they are often designed to cover a broad frequency range, and there are no irregularly shaped mechanical stirrers in SSRCs $[12,13]$. However, SSRCs are generally made of good conductors that cause little loss in the chamber. It often takes a long time for the signal inside to reach the condition of equilibrium, which turns to a huge CPU time for the simulation of SSRCs. Previous studies showed that it costs at least 24 hours for simulating a medium size chamber for just one stirring step on personal computers [14]. Thus accelerating the speed of simulation is one of the challenges in the study of SSRCs.

One way to improve the computational efficiency is introducing additional losses to the chamber [15]. Adding some lossy objects or materials to the chamber can make the electromagnetic signal in RCs attenuate more quickly, but excessive loads in the chamber could degrade the field uniformity and field level, which makes the performance of the chamber unacceptable. Another way to accelerate the simulation of RCs is adding artificial loss to the volume inside the chamber [16], but parameters such as conductivity of the medium inside the cavity used in this method are frequencydependent. If the wide-band result is required, the FDTD iteration has to be conducted repeatedly with the parameters averaged in different narrow bands.

Instead of introducing additional losses in the working volume or on the wall, another method can also be used to accelerate simulation of SSRCs. This method allows us to obtain the frequency response of a nonideal lossy chamber by simulating an ideal lossless chamber and conducting a postprocessing technique on the simulation results. We can obtain a wide-band frequency response of the chamber by running the FDTD iteration just once and subsequently applying the postprocessing at different narrow bands. Although the postprocessing technique was presented in $[17,18]$, there are still some problems. For example, different damping functions are used in these two previous studies and both got the desired results, which is contradictory to each other. In this paper, we give the right form of the damping function. Besides, we first time use the postprocessing technique to simulate the frequency response in a source-stirring chamber and the simulation configuration is specified in detail. In addition, we further discuss and optimize the postprocessing technique in 
terms of the time window and the coefficient in the damping function.

This paper is organized in four sections. The mathematical model of the postprocessing technique and the quality factor ( $Q$ factor) of the chamber is analyzed theoretically in Section 2. The simulation configuration of FDTD method and numerical results of chambers are shown in Section 3. The discussion and optimization of the fast simulation method are described in Section 4.

\section{Mathematical Model}

2.1. The Postprocessing Technique. Suppose a pulse excites the ideal lossless chamber, the chamber can seem to be a system, and an arbitrary scalar electric field $x(t)$ sampled in the working volume of the chamber is the response of the pulse. The corresponding frequency response of the chamber is $X(f)$, which can be written as

$$
X(f)=\sum_{n \in N^{*}} X_{n} \delta\left(f-f_{n}\right),
$$

where $n$ is the index of the resonant frequencies and $N^{*}$ is the set of nature numbers. $X_{n}$ is the coefficient of each resonant frequency and $f_{n}$ denotes the resonant frequencies of the chamber. The resonant frequencies can be also calculated analytically using

$$
f_{m n p}=\frac{c}{2 \pi} \sqrt{\left(\frac{m \pi}{L}\right)^{2}+\left(\frac{n \pi}{W}\right)^{2}+\left(\frac{p \pi}{H}\right)^{2}},
$$

where $L, W$, and $H$ represent the dimensions of the chamber.

Because it is a lossless cavity, the chamber response $x(t)$ will never vanish once the chamber is excited by the pulse and we can not get the frequency response of an endless temporal signal. The response $x(t)$ should be cut by a time window $w(t)$ of duration $T$ and multiplied by a damping function associated with the $Q$ factor of the lossy chamber, which can be written as

$$
x^{\prime}(t)=x(t) w(t) d(t)
$$

where $w(t)$ is a rectangular time window

$$
w(t)= \begin{cases}1, & 0<t \leq T \\ 0, & t>T\end{cases}
$$

and $d(t)$ is a damping exponential function

$$
d(t)=\exp \left(-\frac{\pi f_{0} t}{Q}\right) .
$$

In (5), $f_{0}$ is the center frequency of the narrow bandwidth where we apply the postprocessing technique and value of $Q$ factor is the average value of the quality factor of chamber in the desired frequency band. The Fourier transform of $x^{\prime}(t)$ is

$$
X^{\prime}(f)=\sum_{n \in N^{*}} X_{n} \delta\left(f-f_{n}\right) * W(f) * D(f),
$$

where $D(f)$ is the Fourier transform of the damping function that can be written as

$$
D(f)=\frac{Q}{\pi f_{0}} \frac{1}{1+2 j Q\left(f / f_{0}\right)} .
$$

Suppose $W(f) * D(f)$ equals $D(f)$, which can be indicated in the time domain as $w(t) \cdot d(t) \cong d(t)$, which implies that $d(T) \approx 0$. If we use $d(T)<0.01$ as the criterion, we obtain the restriction of duration of the time window, which can be written as

$$
T>5 \frac{Q}{\pi f_{0}}
$$

and with this restriction, we rewrite (6) as

$$
X^{\prime}(f)=\sum_{n \in N^{*}} X_{n} D\left(f-f_{n}\right)
$$

and substituting (7) for $D(f)$ in (9), we get

$$
X^{\prime}(f)=\sum_{n \in N^{*}} X_{n} \frac{Q}{\pi f_{0}} \frac{1}{1+2 j Q\left(f-f_{n}\right) / f_{0}} .
$$

Since the $Q$ factor of the SSRC varies with frequencies and the value of $Q$ is calculated using the desired center frequency $f_{0}$, this equation is only valid over a narrow frequency band centered at $f_{0}$.

2.2. Calculation of the $Q$ Factor. $Q$ factor is an important parameter measuring the ability of RCs to store the energy. The value of the $Q$ factor of the lossy chamber depends on the inherent loss mechanism of the chamber and the additional loads [15]. It is defined concisely as

$$
Q=\omega_{0} \frac{W_{s}}{P_{d}},
$$

where $\omega_{0}$ is the operating frequency, $W_{s}$ is the energy stored in the chamber, and $P_{d}$ is dissipated power. For a lossy RC without additional loadings, the losses in the chamber walls, antennas, and equipment under test are the dominant loss mechanisms, and they can be approximately calculated as

$$
Q=\frac{3 V}{2 \delta_{w} A} \frac{1}{[1+3 \lambda / 16(1 / L+1 / W+1 / H)]},
$$

where $L, W$, and $H$ are the dimensions of the chamber, $V$ and $A$ are the volume and surface of the cavity, and $\delta_{w}$ represents the skin depth of the cavity wall as

$$
\delta_{w}=\frac{1}{\sqrt{\pi f \sigma_{\mathrm{eff}} \mu}},
$$

where $f$ and $\mu$ are frequency and permeability of the wall. $\sigma_{\text {eff }}$ is the equivalent conductivity of the chamber wall, which means the loss of the chamber wall accounts for all the other loss mechanisms. 


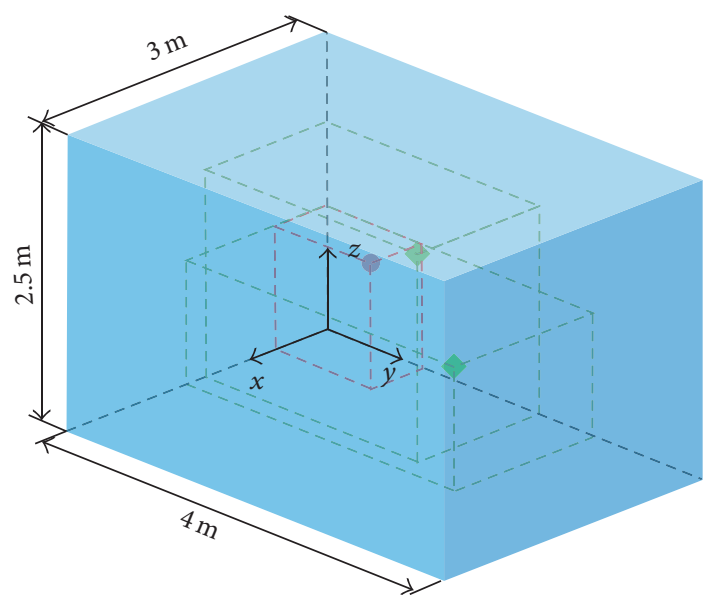

FIGURE 1: The geometry of the source-stirring reverberation chamber.

\section{Simulation of the Chambers}

3.1. Setup and Configuration of the Simulations. Simulations of two chambers are conducted. One is the chamber with PEC walls (lossless chamber), which is also the prototypical chamber employing the postprocessing technique. The other one is a cavity with the walls of good conductors (lossy cavity), which will be used to assess the feasibility of the fast simulation method. In other words, we get and process the temporal signal from the lossless chamber, then transform it to frequency domain, and compared it with the reference frequency signal obtained from the lossy cavity.

The conductivity of the walls of the two chambers is set as $\sigma_{\mathrm{PEC}}=10^{7}$ and $\sigma_{\text {eff }}=5000$, respectively. Except for the conductivity, these two chambers have the same configurations in terms of the dimensions, which are specified as follows: The dimensions of the chambers are $3 \mathrm{~m} \times 4 \mathrm{~m} \times 2.5 \mathrm{~m}$ and the thickness of the chamber wall is $0.025 \mathrm{~m}$. For the sake of simplicity, a dipole antenna operating from $400 \mathrm{MHz}$ to $480 \mathrm{MHz}$ is used as the transmitting antenna. In the Cartesian coordinate system, the dipole is centered at $(0.5,1,1)$ along $y$ axis and excited by a Gaussian pulse, which is indicated by a red dot in Figure 1. Two observation points $\mathrm{P}_{1}(1.5,2.5,1.8)$ and $\mathrm{P}_{2}(2,3,1)$, shown as green diamonds in Figure 1 , are selected to record the electric field during the simulation.

We use a home-made software package CEMS [19], which is based on the FDTD method and is able to run on both CPU and GPU to do the numerical analysis of these two chambers.

3.2. The Time Domain Results. The simulation of the lossless chamber is conducted first. The size of Yee's grid along $x, y$, and $z$ directions is $0.025 \mathrm{~m}$ and the time duration of each step is about 45.7 ps. Because there is no electromagnetic wave propagating out of the chamber, the boundaries on positive/negative $x, y$, and $z$ sides are all PEC. If the total number of simulation steps of the lossless chamber is 1 million, the corresponding signal length is $46 \mu \mathrm{s}$. The electric fields observed at points $\mathrm{P}_{1}$ and $\mathrm{P}_{2}$ are shown in Figure 2.

It is worth noting that the magnitude of the $y$ component is larger than the magnitude of $x$ and $z$ components due
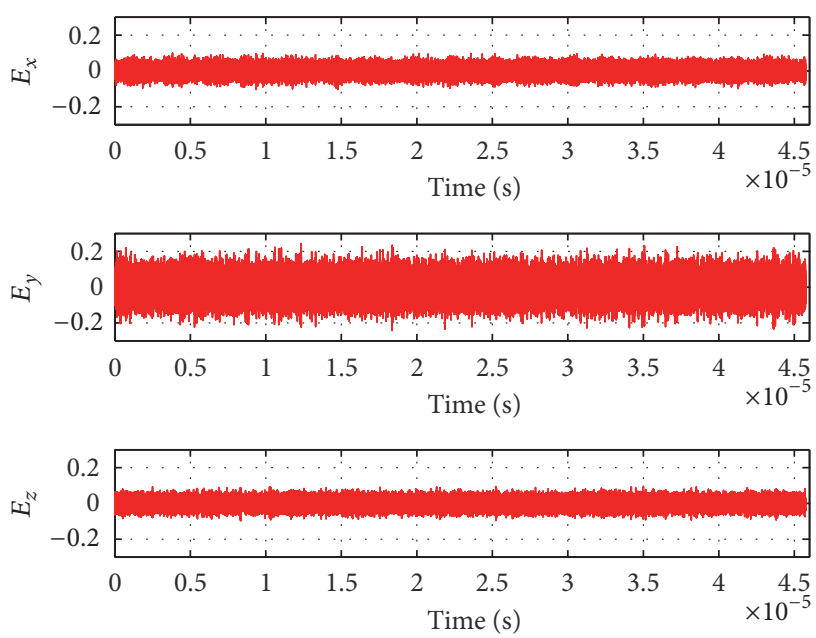

(a)
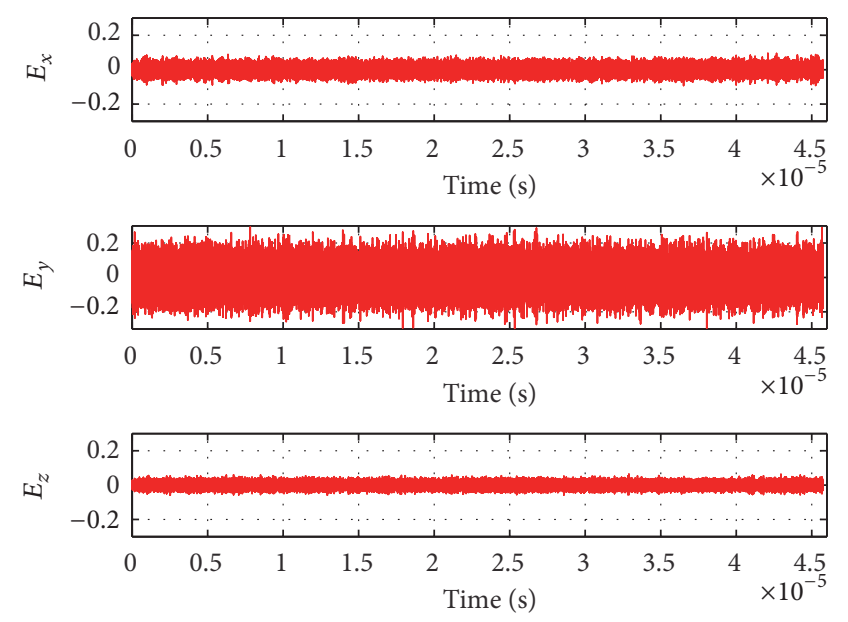

(b)

FIgURE 2: Three components of electric field measured at (a) point 1 and (b) point 2 in the lossless cavity.

to the orientation of the dipole antenna, which is the same as the results of previous studies [7]. We will only discuss the $y$ component of the electric field in the subsequent part of Section 3 and Section 4, since the three components of the electric field have the same characteristics, but the $y$ component is the most dominant.

For the lossy chamber, we use the same mesh and time step for the FDTD iteration. In order to calculate the accurate frequency response of the pulse, we should keep the program running until the field observed attenuates to zero, which needs 8 million iteration steps. This simulation costs the personal computer nearly 15 hours. The $y$ component of electric field measured at $\mathrm{P}_{1}$ and $\mathrm{P}_{2}$ in this lossy chamber is shown in Figure 3. The magnitude of the field is almost zero when the time equals $3.66 \times 10^{-4} \mathrm{~s}$.

\subsection{Implementation of Postprocessing Technique and Fast} Fourier Transform. In order to apply the postprocessing technique to the simulation of SSRCs, some transforms should be done to the original signals obtained in simulation of the 


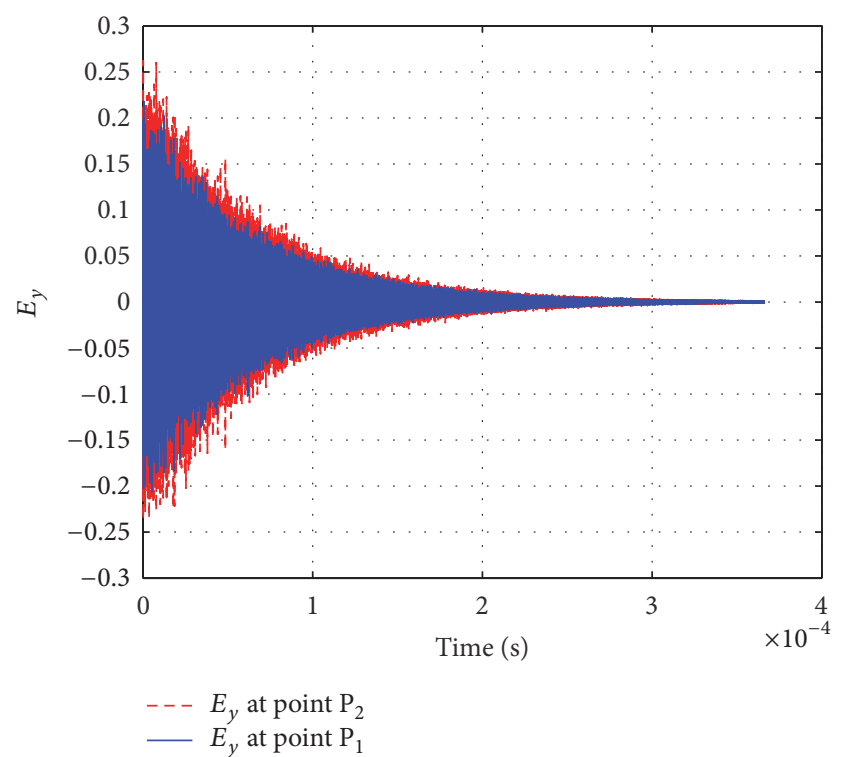

Figure 3: The $y$ components of electric field observed at $\mathrm{P}_{1}$ and $\mathrm{P}_{2}$ in the lossy cavity.

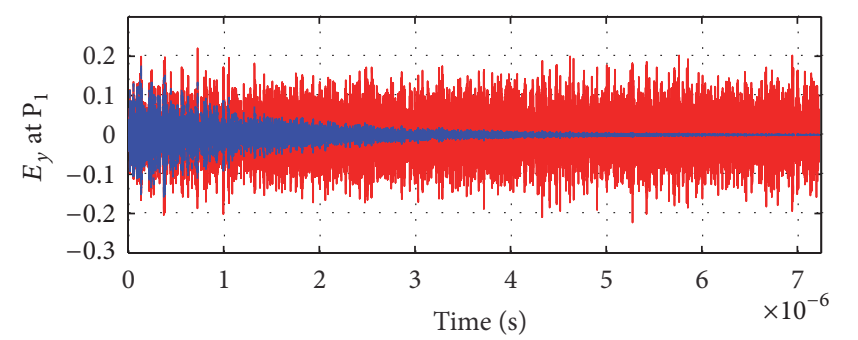

_ Lossless signal

- - Damped signal

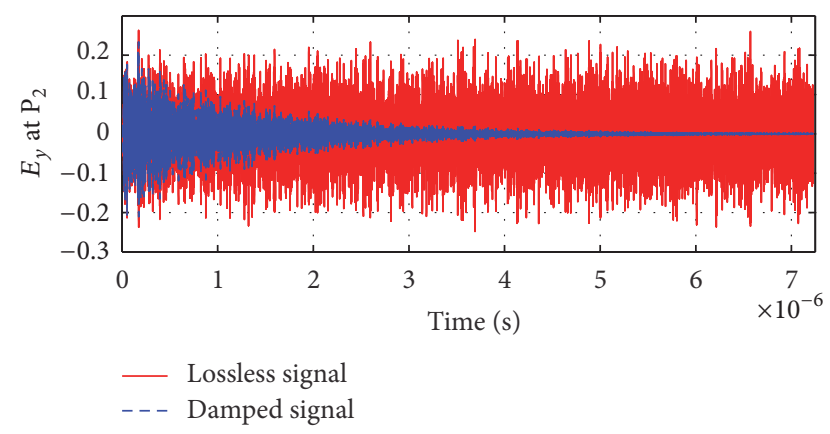

FIgURE 4: The lossless signal and damped signal cut by the time window $w_{1}(t)$.

lossless cavity. The first step to implement the postprocessing technique is to add a time window $w(t)$ and damping function $d(t)$ to the electric field signal. The damping function is presented in (5) and the length of the time window can be determined by (8). The $Q$ factor in these two functions is 1996 at frequency $f_{0}=440 \mathrm{MHz}$, which is calculated via (11) and (12). When the time window $w_{1}(t)=7.2 \mathrm{~ms}$, the lossless signal and damped signal are shown in Figure 4.

The time for the signal attenuating to zero in the simulation of the lossy cavity is much longer than the time window
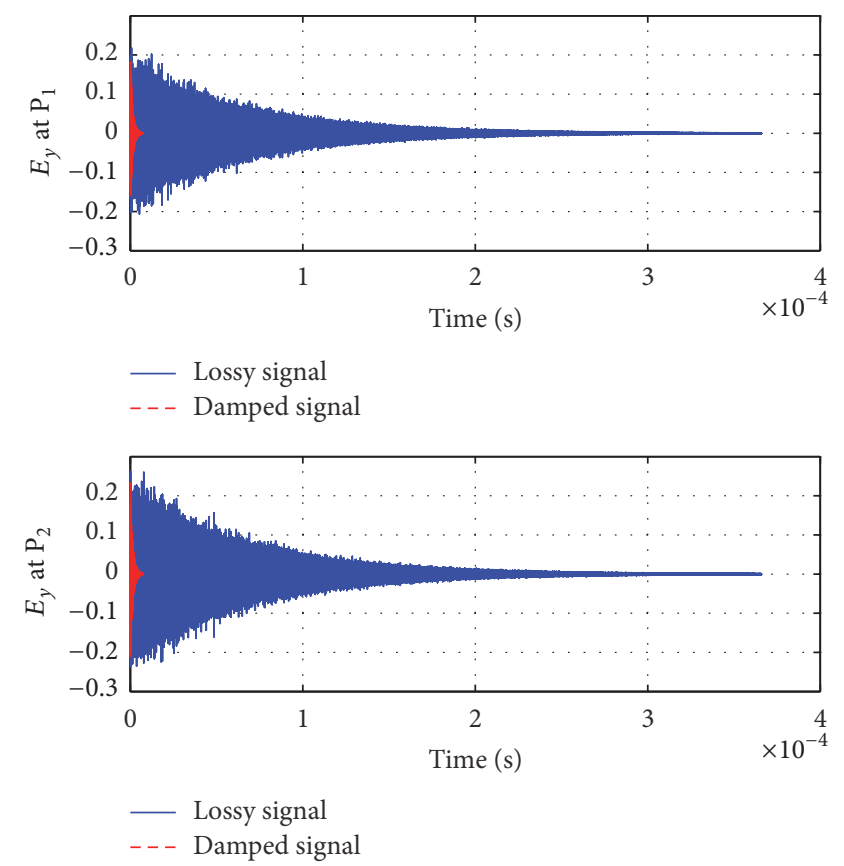

FIGURE 5: The comparison of the damped signal and attenuating signal. The time window of the damped signal is $w_{1}(t)$.

$w_{1}(t)$ used to cut the lossless signal, and the comparison of the attenuating signal from lossy cavity and damped signal is shown in Figure 5.

From the comparison we can see that the length of the lossy signal is at least 50 times longer than that of the damped signal from the lossless chamber, which means large amount of simulation time is saved. It is very important for the simulation of the SSRC because the simulation of the chamber must be conducted for enough "stirring steps" to get the desired statistically uniform electromagnetic environment inside the cavity.

The fast Fourier transform (FFT) of the damped signal and the attenuating signal from the lossy chamber is performed. Since the frequency response of the damped signal can only be valid around the center frequency $f_{0}=440 \mathrm{MHz}$ and the operating frequency of the dipole antenna is from $400 \mathrm{MHz}$ to $480 \mathrm{MHz}$, therefore we choose to do the FFT from $420 \mathrm{MHz}$ to $460 \mathrm{MHz}$.

Figure 6 shows that the frequency waveform of the damped signal, which is postprocessed, does not match that of attenuated signal from the lossy cavity very well especially at some frequencies where the peaks exist. Thus, we further discuss various conditions of the fast simulation method of the SSRCs.

\section{Optimization of the Postprocessing Technique}

First, different length of time window is chosen to see whether the change of the time window could improve the result of postprocessing technique. Suppose we set the length of the new time window $w_{2}(t)=21.6 \mu \mathrm{s}$, which is three times as 

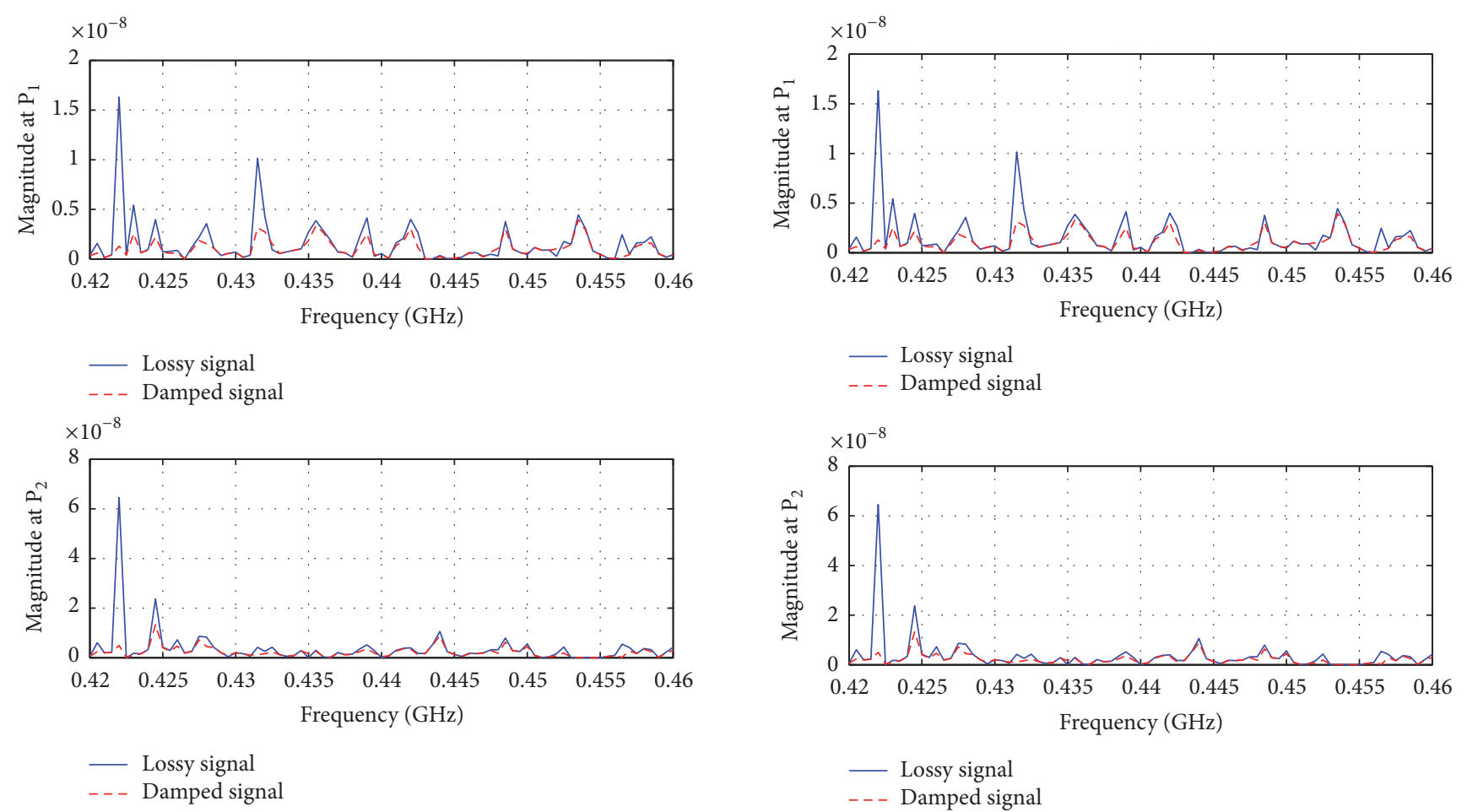

FIgURE 6: The FFT of the processed signal and lossy signal.
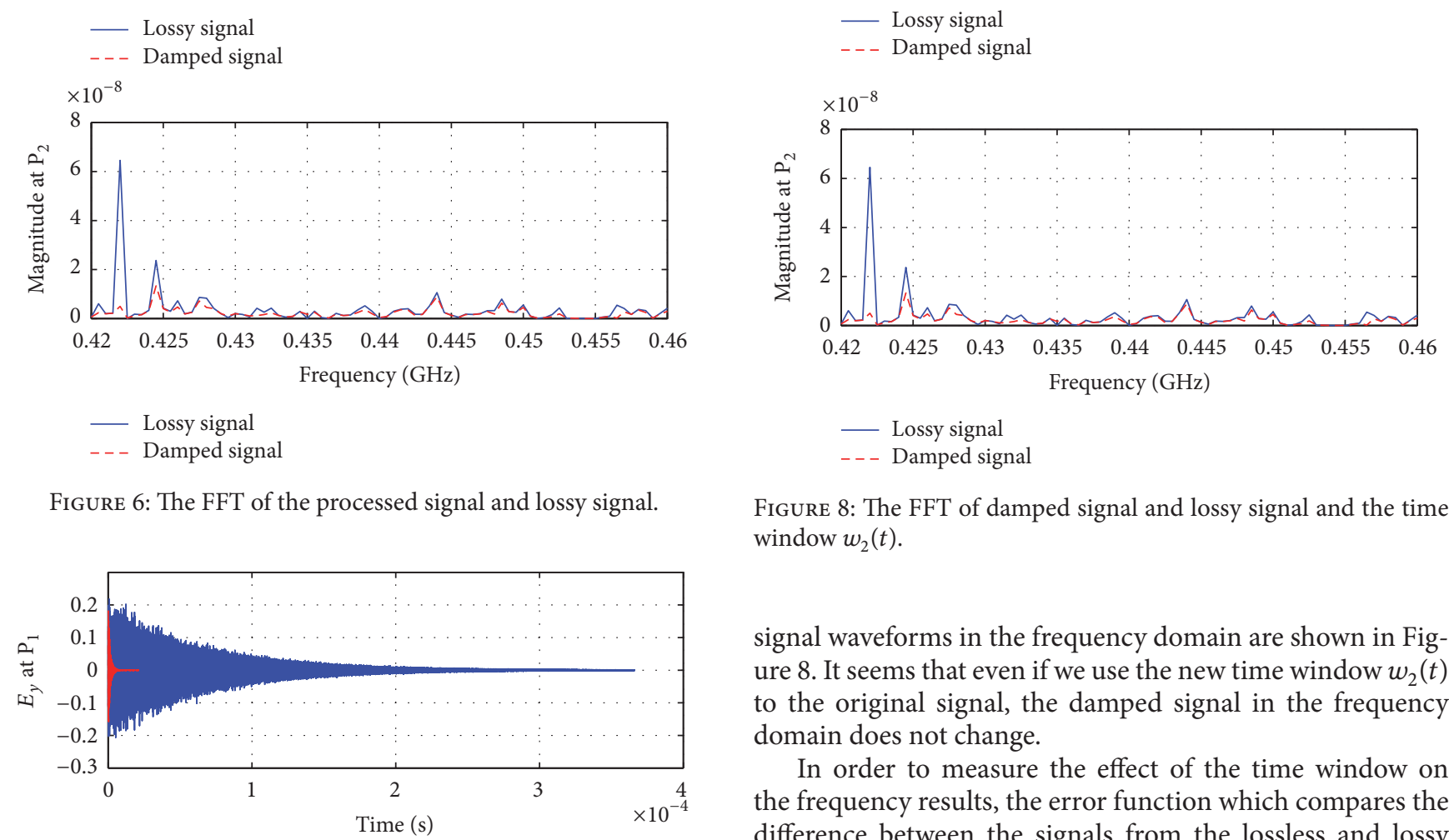

FIgUrE 8: The FFT of damped signal and lossy signal and the time window $w_{2}(t)$.

signal waveforms in the frequency domain are shown in Figure 8 . It seems that even if we use the new time window $w_{2}(t)$ to the original signal, the damped signal in the frequency domain does not change.

In order to measure the effect of the time window on the frequency results, the error function which compares the difference between the signals from the lossless and lossy cavity is defined as

$$
\operatorname{err}(f)=\left|X_{\text {damped }}(f)-X_{\text {lossy }}(f)\right|,
$$

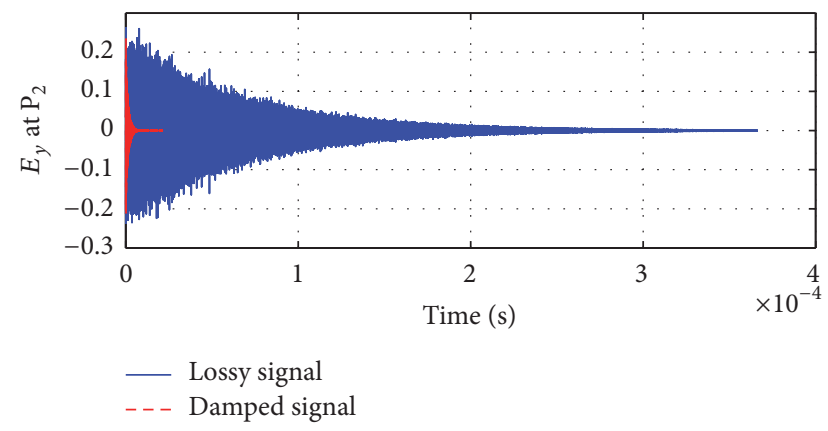

FIgURE 7: The comparison of the damped signal and attenuating signal and the time window $w_{2}(t)$.

long as $w_{1}(t)$. The original signal obtained from the lossless cavity is cut by time window $w_{2}(t)$ and damped by the function in (5). Then we get the damped signal cut by time window $w_{2}(t)$ in the time domain in Figure 7 , compared with the lossy signal obtained in the lossy chamber. The corresponding

where $X_{\text {damped }}(f)$ and $X_{\text {lossy }}(f)$ is the frequency waveform of damped signal and lossy signal, respectively.

The errors of the waveform in the frequency domain in the case of the time windows $w_{1}(t)$ and $w_{2}(t)$ are shown in Figure 9. The error waveforms are the same in these two cases, which also proves that the change of the time window has no effect on the frequency results from the lossless cavity.

In the second attempt, a coefficient $\rho$ is introduced to the damping function $d(t)$. We add the coefficient $\rho$ to (5), which can be rewritten as

$$
d(t)=\exp \left(-\frac{\rho \pi f_{0} t}{Q}\right) .
$$

If the value of the coefficient $\rho=1 / 3$, and the proper length of time window $w(t)=w_{2}(t)$ is chosen here, the damped signal and lossy signal in the time domain are shown in Figure 10, and the frequency waveforms of these two signals are shown in Figure 11.

Figure 11 illustrates that the introduction of the coefficient $\rho$ improves the frequency waveform on the basis of the time 

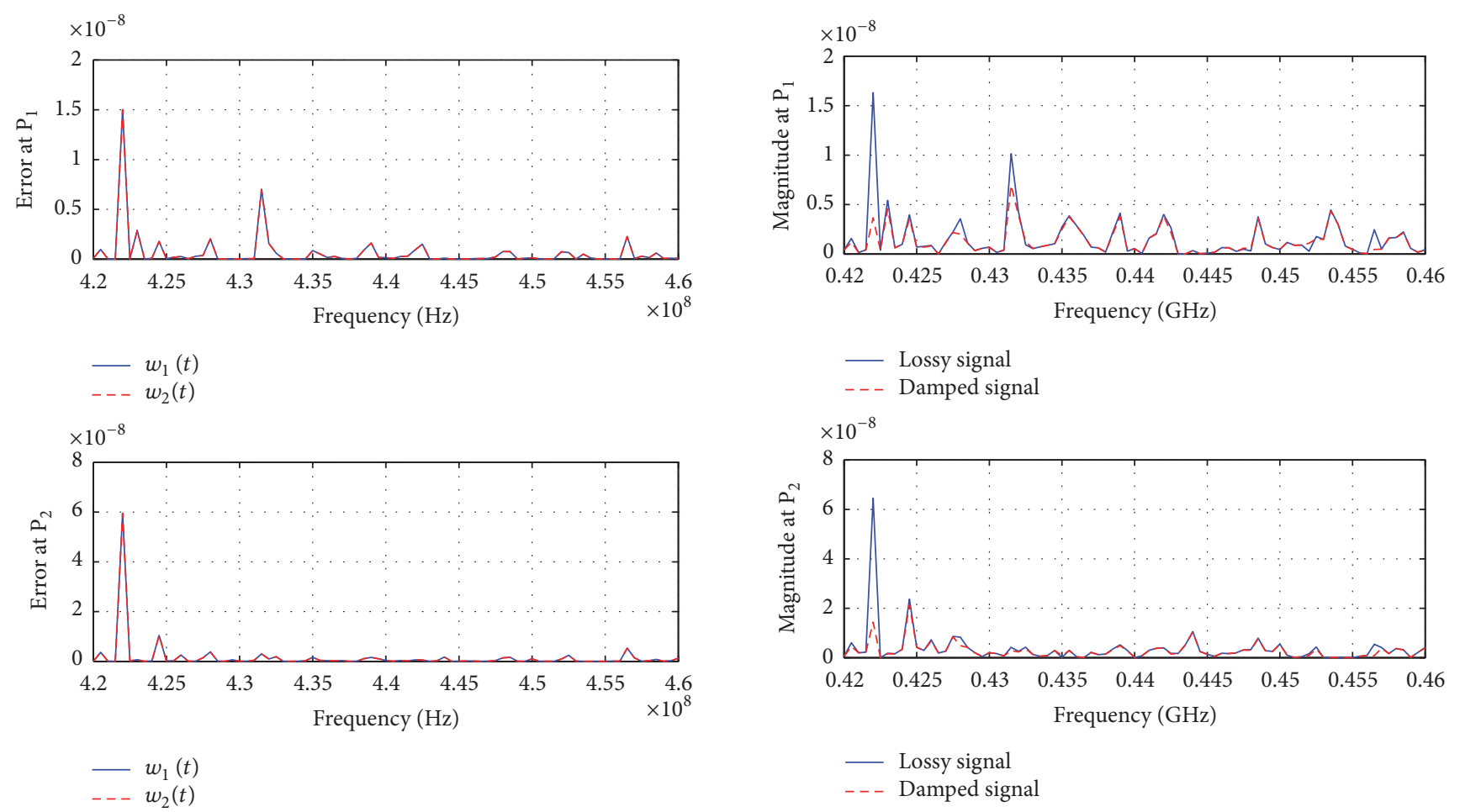

FIGURE 9: The waveform of error for the cases of $w_{1}(t)$ and $w_{2}(t)$.
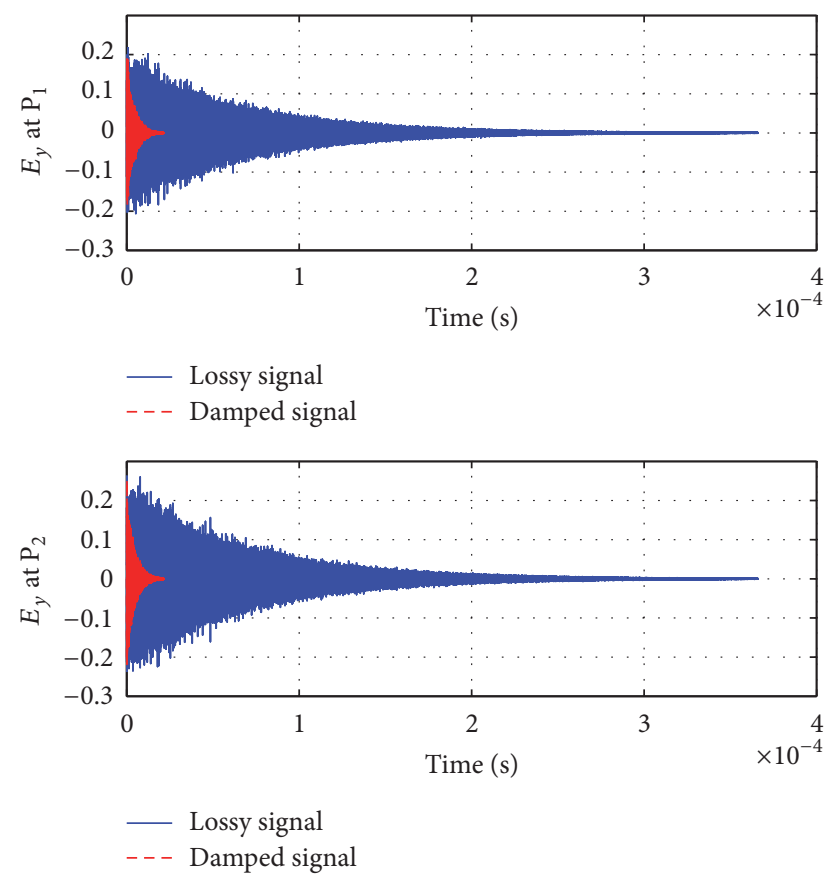

FIgURE 10: The comparison of the damped signal and lossy signal. Time window is $w_{2}(t)$ and the coefficient is $\rho=1 / 3$.

window $w_{2}(t)$. To further improve the results, the simulation is also conducted under the condition of time window $w_{3}(t)=$ $35.6 \mu \mathrm{s}$, and the coefficient $\rho=0.2$. The error functions $\operatorname{err}(f)$ of this condition, together with the condition of $w_{2}(t), \rho=$ $1 / 3$ are compared in Figure 12, which shows that the $\operatorname{err}(f)$

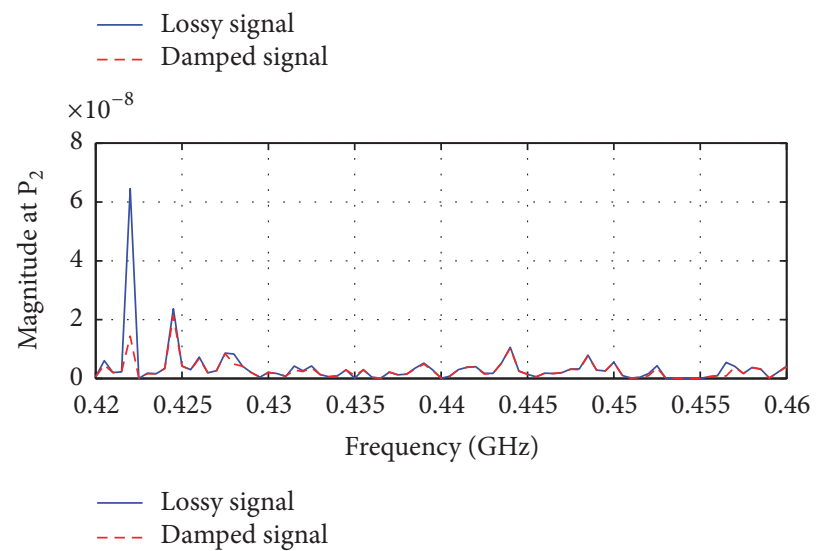

FIGURE 11: The FFT of damped signal and lossy signal. Time window is $w_{2}(t)$ and the coefficient is $\rho=1 / 3$.

decreases to almost zero over the whole frequency range in the case of $w_{3}(t)$ and $\rho=0.2$, which means this condition gives the best match of the damped signal (obtained in the lossless cavity and postprocessed) to the reference lossy signal (obtained in the lossy chamber) in the frequency domain.

The length of the time window should be chosen depending on the purpose of the simulation of the chamber. The time window should be larger if one is interested in the accurate field inside the chamber, and for other purposes such as the optimization of the transmitting antenna, the minimum time window can be used.

The postprocessing technique allows us to obtain the frequency response of the real lossy chamber via the FDTD iteration of the lossless chamber. The simulation time of this method is also very short compared to traditional simulation method of SSRCs. All the simulations are run on both a personal computer (GPU: GeForce GTX $750 \mathrm{Ti}, 2 \mathrm{G}$ Ram) and a server (GPU: GeForce GTX TitanX, 12 G Ram). The iteration steps, the length of the signal, and the simulation time of lossless cavity in terms of different cases and the lossy cavity are shown in Table 1. The time spent on the FDTD iteration of the lossless cavity on a PC is much shorter than the time of the lossy cavity. The simulation time is even shorter if we conduct the simulation on a server. The short simulation time of the SSRCs can greatly improve the efficiency since the simulation should be run for dozens of times at each stirring step to get the accurate statistical characteristic of the chamber. And this fast simulation method makes it possible to run the full wave simulation of SSRCs on a PC. 
TABLE 1: The simulation time of FDTD iteration of the lossy chamber and lossless chamber in the case of different time windows.

\begin{tabular}{lcccc}
\hline & FDTD steps & Signal length & Simulation time (PC) & Simulation time (server) \\
\hline Lossy cavity & $8.00 * 10^{6}$ & $365.91 \mu \mathrm{s}$ & $89 \mathrm{~h} 1 \mathrm{~m}$ & $14 \mathrm{~h} 44 \mathrm{~m}$ \\
Lossless cavity 1 & $1.58 * 10^{5}$ & $7.23 \mu \mathrm{s}$ & $23 \mathrm{~m} 2 \mathrm{~s}$ & $37 \mathrm{~s}$ \\
Lossless cavity 2 & $4.74 * 10^{5}$ & $21.68 \mu \mathrm{s}$ & $1 \mathrm{~h} 9 \mathrm{~m}$ & $1 \mathrm{~m} 51 \mathrm{~s}$ \\
Lossless cavity 3 & $7.90 * 10^{5}$ & $36.14 \mu \mathrm{s}$ & $1 \mathrm{~h} 55 \mathrm{~m}$ & $2 \mathrm{~m} 17 \mathrm{~s}$ \\
\hline
\end{tabular}
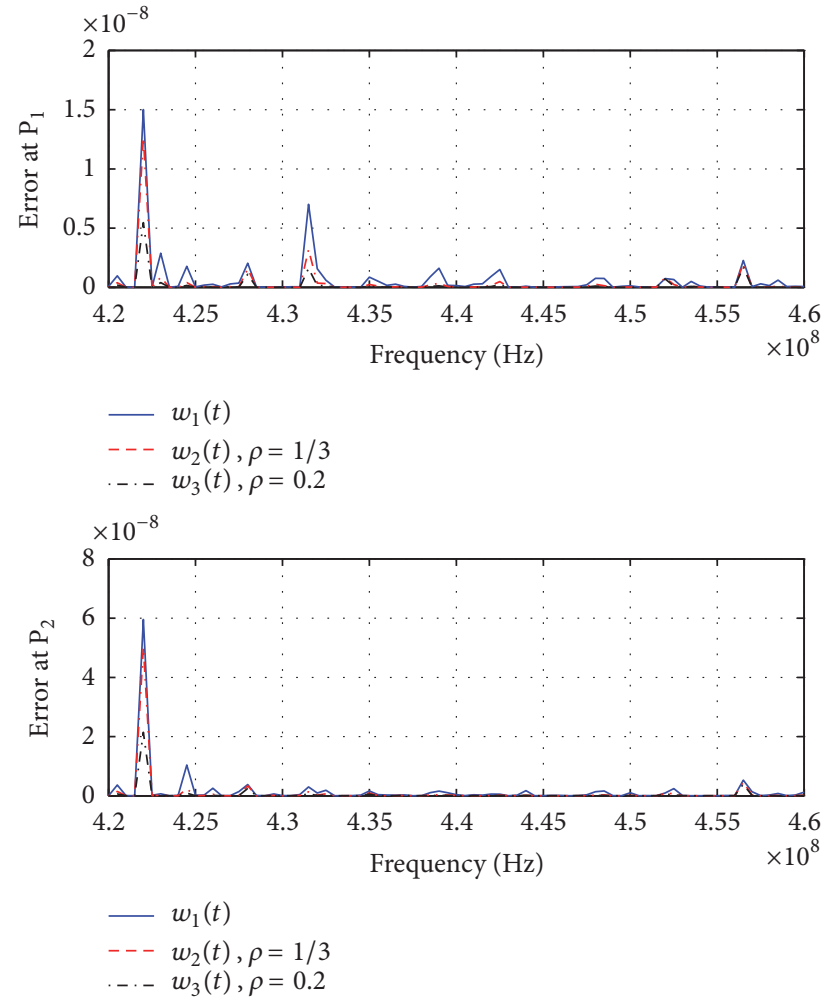

FIGURE 12: The waveform of error in case of different time window $w(t)$ and coefficient $\rho$.

\section{Conclusion}

The fast simulation method of the SSRC is presented in this paper. In this method, one does not have to wait long for the state of equilibrium to get the frequency response of a lossy chamber. To implement this method, the FDTD iteration of a lossless chamber and the postprocessing technique of the FDTD results are required. The theoretical model of the postprocessing technique, the $Q$ factor used to represent the loss mechanism of the lossy chamber, and the setup configuration of the FDTD method are all introduced.

In order to verify this fast simulation method, both a lossy and a lossless cavity are simulated. The simulation results of these two SSRCs are given in detail, which show that the fast simulation method can achieve the good frequency response of the chamber and reduce the simulation time dramatically. Although the frequency response is obtained in a narrow band in this paper, we can get the frequency response for a wider band by applying this method to other center frequencies. More importantly, repetitive FDTD iteration is not needed to expand the frequency results. The further discussion of the postprocessing technique shows that proper application of time window and coefficient in the damping function can further improve the accuracy of frequency waveform of the simulation results.

\section{Competing Interests}

The authors declare that there is no conflict of interests regarding the publication of this paper.

\section{References}

[1] Q. Xu, Y. Huang, S. Yuan, L. Xing, and Z. Tian, "Two alternative methods to measure the radiated emission in a reverberation chamber," International Journal of Antennas and Propagation, vol. 2016, Article ID 5291072, 7 pages, 2016.

[2] J. Anguera, A. Andújar, M.-C. Huynh, C. Orlenius, C. Picher, and C. Puente, "Advances in antenna technology for wireless handheld devices," International Journal of Antennas and Propagation, vol. 2013, Article ID 838364, 25 pages, 2013.

[3] IEC, "Electromagnetic compatibility (EMC) - part 4-21: testing and measurement techniques-reverberation chamber test methods," IEC 61000-4-21, 2011.

[4] P. Corona, J. Ladbury, and G. Latmiral, "Reverberationchamber research-then and now: a review of early work and comparison with current understanding," IEEE Transactions on Electromagnetic Compatibility, vol. 44, no. 1, pp. 87-94, 2002.

[5] Y. Huang and D. J. Edwards, "Novel reverberating chamber: the source-stirred chamber," in Proceedings of the 8th International Conference on Electromagnetic Compatibility, September 1992.

[6] V. Mariani Primiani, P. Russo, and G. Cerri, "Experimental characterization of a reverberation chamber excited by the source stirring technique," in Proceedings of the International Symposium on Electromagnetic Compatibility (EMC EUROPE '12), pp. 1-6, September 2012.

[7] G. Cerri, C. D. Sanctis, V. M. Primiani, C. Monteverde, and P. Russo, "Array of antennas design for the source stirring technique," in Proceedings of the 20th International Zurich Symposium on Electromagnetic Compatibility, pp. 105-108, IEEE, Zurich, Switzerland, January 2009.

[8] W. X. Li, C. Y. Yue, and W. H. Yu, "Study on array source stirring reverberation chamber," Applied Computational Electromagnetics Society Journal, vol. 29, no. 12, pp. 1067-1076, 2014.

[9] K. Harima and Y. Yamanaka, "FDTD analysis on the effect of stirrers in a reverberation chamber," in Proceedings of the 4th International Symposium on Electromagnetic Compatability, pp. 260-263, Tokyo, Japan, 1999.

[10] J. S. Kim and R. Mittra, "Performance evaluation of a modestirred reverberation chamber using the finite difference time domain (FDTD) simulation," in Proceedings of the Asia-Pacific 
Symposium on Electromagnetic Compatibility, pp. 173-176, Singapore, May 2012.

[11] F. Moglie and A. P. Pastore, "FDTD analysis of plane wave superposition to simulate susceptibility tests in reverberation chambers," IEEE Transactions on Electromagnetic Compatibility, vol. 48, no. 1, pp. 195-202, 2006.

[12] G. Cerri, V. M. Primiani, and P. Russo, "Reverberation chamber field modeling for application to the source stirring technique," in Proceedings of the Asia-Pacific International Symposium on Electromagnetic Compatibility (APEMC '10), pp. 536-539, IEEE, Beijing, China, April 2010.

[13] G. Cerri, V. M. Primiani, C. Monteverde, and P. Russo, "A theoretical feasibility study of a source stirring reverberation chamber," IEEE Transactions on Electromagnetic Compatibility, vol. 51, no. 1, pp. 3-11, 2009.

[14] F. Moglie, "Convergence of the reverberation chambers to the equilibrium analyzed with the finite-difference time-domain algorithm," IEEE Transactions on Electromagnetic Compatibility, vol. 46, no. 3, pp. 469-476, 2004.

[15] C. L. Holloway, D. A. Hill, J. M. Ladbury, and G. Koepke, "Requirements for an effective reverberation chamber: unloaded or loaded," IEEE Transactions on Electromagnetic Compatibility, vol. 48, no. 1, pp. 187-194, 2006.

[16] Y. Zhao, G. Wei, Y. Cui, L. Fan, X. Pan, and H. Wan, "Acceleration technique of modeling lossy reverberation chamber using FDTD method based on quality factor," IEEE Antennas and Wireless Propagation Letters, vol. 14, pp. 686-689, 2015.

[17] G. Orjubin, F. Petit, E. Richalot, S. Mengué, and O. Picon, "Cavity losses modeling using lossless FDTD method," IEEE Transactions on Electromagnetic Compatibility, vol. 48, no. 2, pp. 429-431, 2006.

[18] P. Bonnet, R. Vernet, S. Girard, and F. Paladian, "FDTD modelling of reverberation chamber," Electronics Letters, vol. 41, no. 20, pp. 1101-1102, 2005.

[19] A. Z. Elsherbeni and V. Demir, The Finite-Difference TimeDomain Method for Electromagnetics with MATLAB Simulations, SciTech Publishing, Edison, NJ, USA, 2nd edition, 2016. 


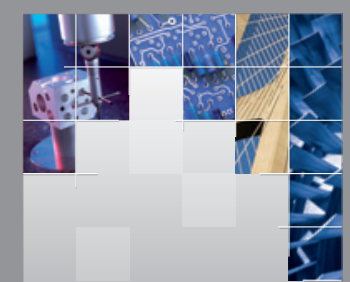

\section{Enfincering}
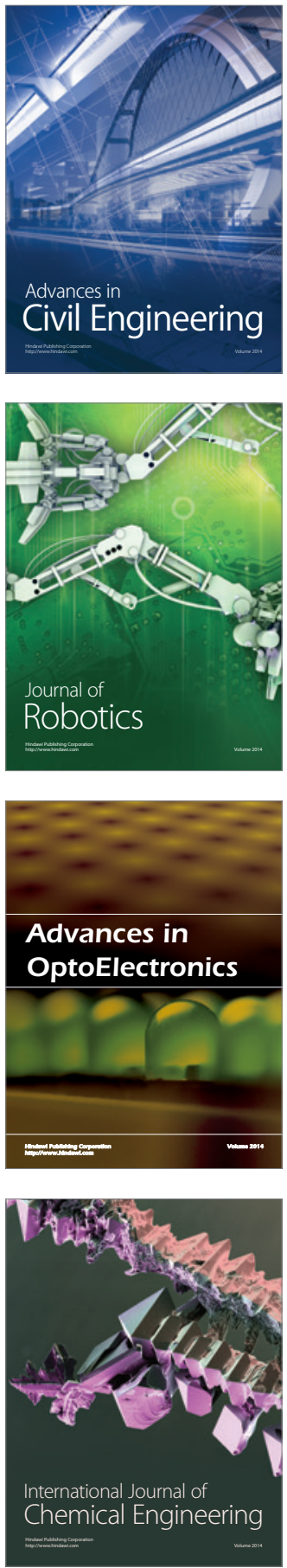

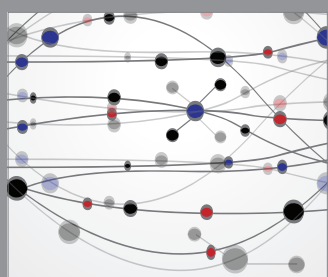

The Scientific World Journal

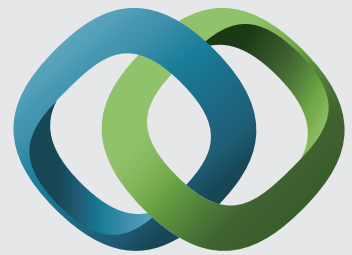

\section{Hindawi}

Submit your manuscripts at

https://www.hindawi.com
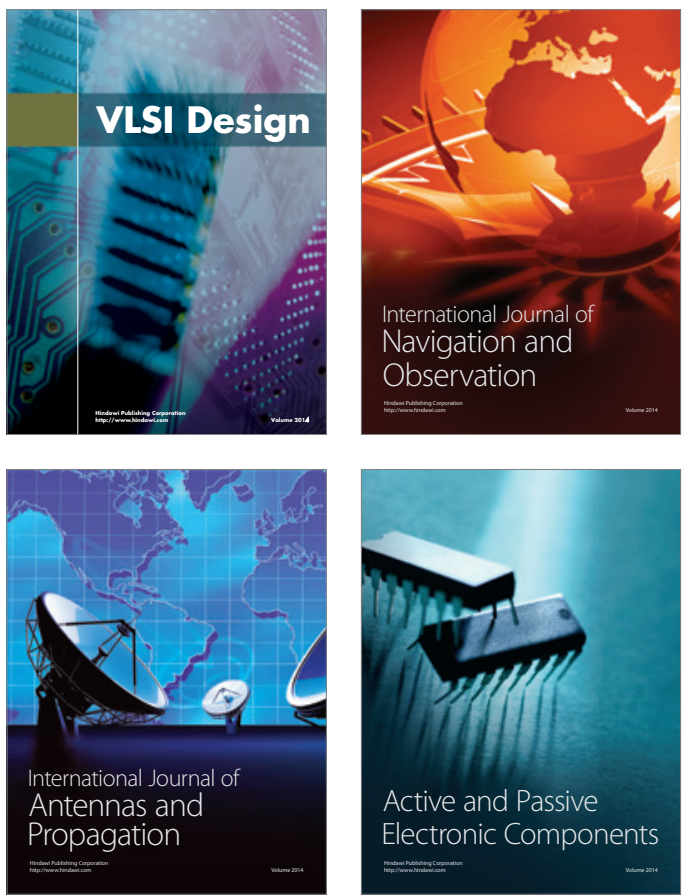
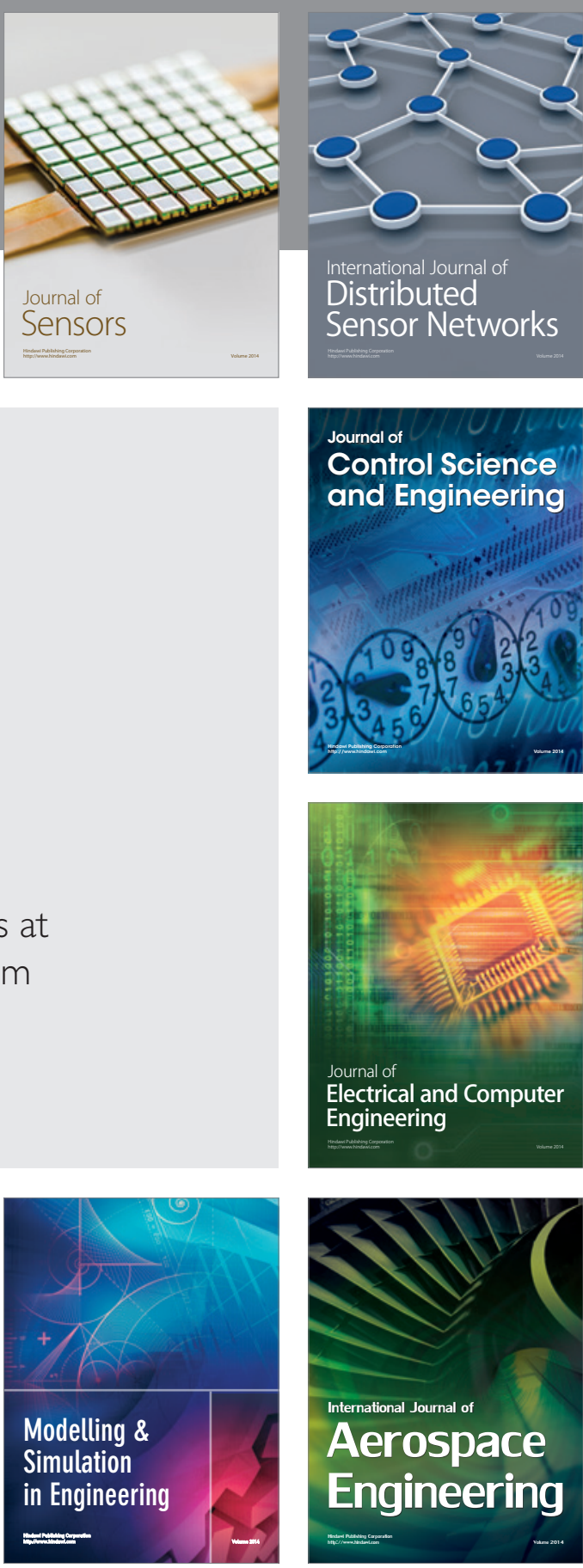

International Journal of

Distributed

Sensor Networks

$-$

Joumal of

Control Science

and Engineering
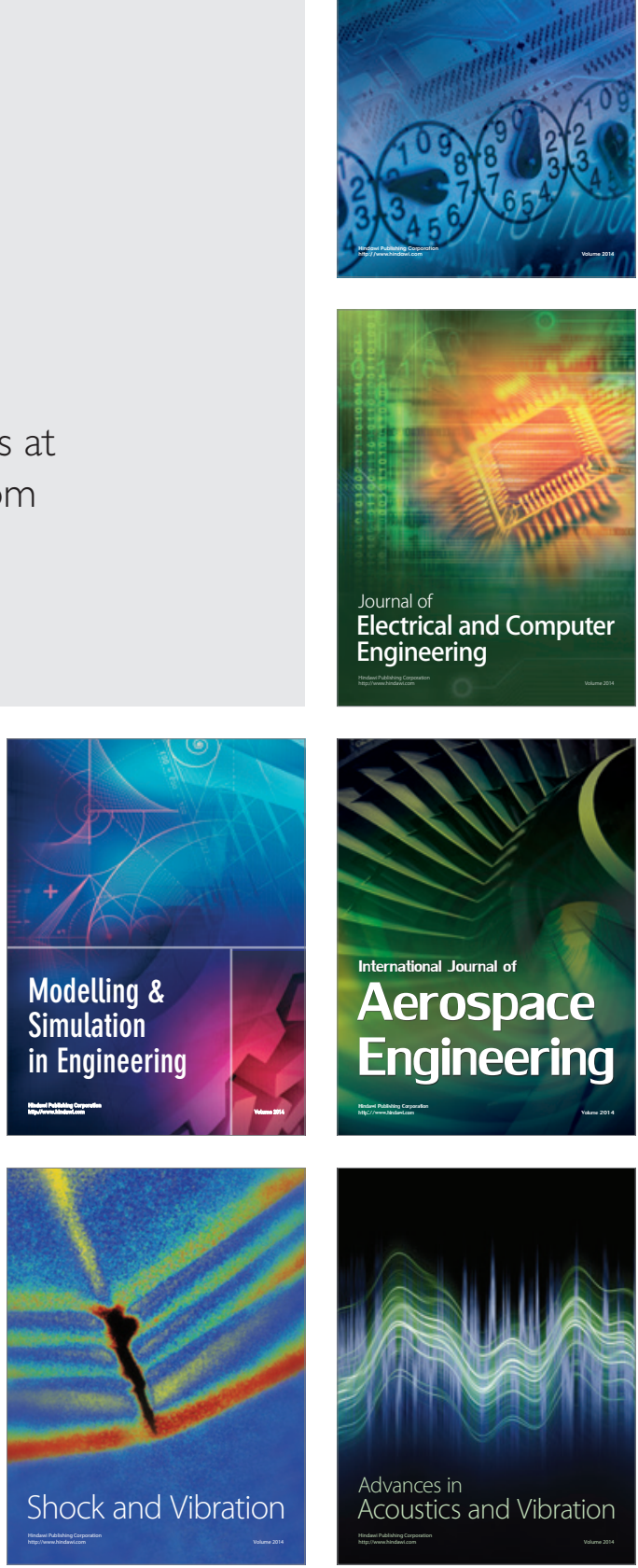